\title{
Hybrid Quadrilateral Finite Element Models for Axial Symmetric Helmholtz Problem
}

\author{
K.Y.Sze ${ }^{1 *}$, Q.H.Zhang ${ }^{1,2}$, G.H.Liu ${ }^{1}$ \\ ${ }^{1}$ Department of Mechanical Engineering, The University of Hong Kong, Pokfulam, Hong Kong SAR, P.R.China. \\ ${ }^{2}$ Department of Scientific Computing \& Computer Applications, Sun Yat-Sen University, Guangzhou, P.R. China.
}

*Correspondence author (Email: kysze@hku.hk)

\begin{abstract}
This paper is a continuation of the previous work in which six-node triangular finite element models for the axial symmetric Helmholtz problem are devised by using a hybrid functional and the spherical-wave modes [1]. The six-node models can readily be incorporated into the standard finite element program framework and are typically $\sim 50 \%$ less erroneous than their conventional or, equivalently, continuous Galerkin counterpart. In this paper, four-node and eight-node quadrilateral models are devised. Two ways of selecting the spherical-wave modes are attempted. In the first way, a spherical-wave pole is selected such that it is equal-distant from an opposing pair of element nodes. In the second way, the directions of the spherical-waves passing through the element origin are equal-spaced with one of the directions bisecting the two parametric axes of the element. Examples show that both ways lead to elements that yield very similar predictions. Furthermore, four-node and eight-node hybrid elements are typically $\sim 50 \%$ and $\sim 70 \%$ less erroneous than their conventional counterparts, respectively.
\end{abstract}

Keywords: axial symmetric; Helmholtz; hybrid; spherical-wave; finite element

Published in Finite Elements in Design \& Analysis 52: 1-10 (2012). doi:10.1016/j.finel.2011.12.001 


\section{INTROUDCTION}

A major challenge in finite element analyses of Helmholtz problems is that the solutions are spatially oscillating throughout the entire problem domains. This contrasts sharply with stress analyses in which high displacement gradients only occur in the stress concentration regions. While considerable computational saving can be realized by using graded meshes in stress analyses, the practice is not applicable to Helmholtz problems. Hence, the mesh requirement induces tremendous computing load when the wavenumber or the problem domain size increases. To better tackle the issue, a number of wave-based approaches that make use of the solution sets for the wave or Helmholtz equations have been proposed in the last decades. These include the Trefftz methods [1-9], the partition of unity method [10-13], the discontinuous Galerkin method [14,15], among others.

Among the Trefftz finite element models, the least-square models [2,3], the traction-frame models [4] and the displacement-frame models [1,6-9] can be noted. All Trefftz models possess their own discontinuous Helmholtz solution modes or, simply, discontinuous modes which satisfy the Helmholtz equation strictly. The least-square models are formulated by minimizing a weighted sum of the error norms of the boundary and continuity conditions on the discontinuous modes. The traction-frame and displacement-frame models are hybrid models which are also equipped with boundary variables equivalent to "traction" and "displacement" in elasticity [16], respectively, whilst the discontinuous modes can be condensed at the element level.

In the partition of unity finite element method, the plane-wave solutions are employed as the nodal enrichment functions [10-13]. The value of the Helmholtz variable at a node is the sum of plane-wave solutions which represent plane-waves propagating along different directions. Within the element, the Helmholtz variable is obtained by the conventional nodal interpolation. Thus, the system equation unknowns are the amplitudes of the plane-waves at the nodes but not the nodal value of the Helmholtz variable.

In the discontinuous Galerkin or enrichment method, the nodal interpolation is enhanced by enrichment functions as in the incompatible element formulation [14,15]. The enrichment functions induce discontinuity across the element boundary and enforcement of the continuity is implemented through Lagrange multipliers. While the enrichment functions can be condensed at element level, the multipliers which link the enrichments of adjacent elements enter the global equation.

This paper is a continuation of the previous work in which six-node hybrid "displacement-frame" models for the axial symmetric Helmholtz problem are devised by using the spherical-wave solution to construct the basis of the discontinuous modes [1]. Here, four-node and eight-node quadrilateral models are devised by using two different sets of spherical-wave modes. In the first way, a spherical- 
wave pole is selected such that it is equal-distant from an opposing pair of element nodes. In the second way, the directions of the spherical-waves passing through the element origin are equalspaced with one of the directions bisecting the two parametric axes of the element. Examples show that elements employing "equal-distant" and "equal-spaced" wave modes produce very similar predictions. The errors of the four-node and eight-node hybrid elements are respectively $\sim 50 \%$ and $\sim 70 \%$ less erroneous than their conventional counterparts. In particular, the hybrid models can readily be plugged into any standard finite element program .

\section{CONVENTIONAL AND HYBRID FORMULATIONS}

In the computational community of Helmholtz and related problems, the conventional finite element formulation is often known as the continuous Galerkin formulation which simply means that the $\mathrm{C}^{0}$ node-based trial functions are used as the weight functions. The variational arguments for the conventional and the hybrid formulations to be employed have been presented in reference [1,6-9]. To save space, only a very brief summary will be provided here. This paper will restrict itself to bounded domains. Under the axial symmetry described by coordinates $(r, z)$, Helmholtz equation is [1]:

$$
\left[\frac{1}{r} \frac{\partial}{\partial r}\left(r \frac{\partial}{\partial r}\right)+\frac{\partial^{2}}{\partial z^{2}}+k^{2}\right] u=0
$$

and the element functional for the conventional formulation can be expressed as :

$$
\Pi^{e}=\frac{1}{2} \int_{A^{e}}\left(u,{ }_{r} u,{ }_{r}+u,,_{z} u,_{z}-k^{2} u^{2}\right) r d A-\int_{\Gamma_{t}^{e}} \bar{t} u r d \Gamma
$$

where ()${ }_{r}=\partial() / \partial r,()_{, z}=\partial() / \partial z, A^{e}$ is the element area in the $r$-z-plane and $u$ obtained by nodal interpolation is $\mathrm{C}^{0}$ across the element boundary. $\Gamma_{t}^{e}$ is the element boundary in the $r$ - $z$-plane prescribed with the natural boundary condition $n_{r} u,{ }_{r}+n_{z} u,,_{z}=\bar{t}$ in which $\left\{n_{r}, n_{z}\right\}^{T}=\left\{\cos \theta_{n}, \sin \theta_{n}\right\}^{T}$ is the unit outward normal vector to the element boundary, see Figure 1. The following elemental hybrid functional can be formed by introducing another Helmholtz variable $g$ which is discontinuous across the element boundary to (2) as [1]:

$$
\Pi_{h}^{e}=\frac{1}{2} \int_{A^{e}}\left(g, r g,{ }_{r}+g,_{z} g,,_{z}-k^{2} g^{2}\right) r d A+\int_{\partial A^{e}}\left(n_{r} g,{ }_{r}+n_{z} g,_{z}\right)(u-g) r d \Gamma-\int_{\Gamma_{t}^{e}} \bar{t} u r d \Gamma
$$

where $\partial A^{e}$ denotes the complete boundary of $A^{e}$. It is trivial to see that second integral enforces the equality of the $u$ and $g$ over $\partial A^{e}$. When $g$ satisfies (1), the above functional can be re-written as: 


$$
\Pi_{h}^{e}=\int_{\partial A^{e}}\left[-\frac{1}{2}\left(n_{r} g,{ }_{r}+n_{z} g,{ }_{z}\right) g+\left(n_{r} g,{ }_{r}+n_{z} g,{ }_{z}\right) u\right] r d \Gamma-\int_{\Gamma_{t}^{e}} \bar{t} u \cdot r d \Gamma
$$

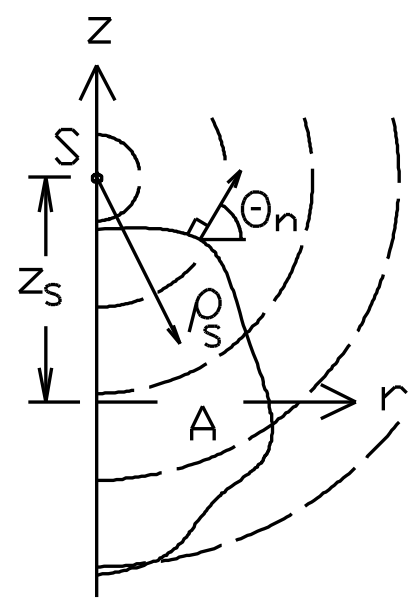

Figure 1. Cross section A of an axial symmetric body in the r-z-plane and the spherical-wave $u=$ $\exp \left(i k \rho_{s}\right) /\left(k \rho_{s}\right)$, where $\rho_{s}^{2}=r^{2}+\left(z-z_{s}\right)^{2}$, from the pole $\mathrm{S}\left(0, z_{s}\right)$. Over the boundary of A, $\theta_{n}$ denotes the inclination of the outward normal vector to the $r$-axis.

which involves only boundary integral. For a $n$-node element, the continuous variable $u$ can be expressed as:

$$
u=\sum_{i=1}^{n} N_{i} u_{i}=\left[N_{1}, \ldots, N_{n}\right]\left\{\begin{array}{c}
u_{1} \\
\vdots \\
u_{n}
\end{array}\right\}=\mathbf{N d}
$$

in which $N_{i}=N_{i}(\xi, \eta)$ is the nodal interpolation function, $(\xi, \eta)$ are the parametric coordinates and $u_{i}$ is the nodal value of $u$. Other terms are self-defined. The discontinuous variable $g$ can be expressed as:

$$
g=\mathbf{P} \boldsymbol{\beta}
$$

where $\mathbf{P}$ is the shape function matrix and $\boldsymbol{\beta}$ is the vector of coefficients. With the last two equations, the functionals in (2) and (4) become

$$
\Pi^{e}=\frac{1}{2} \mathbf{d}^{T} \mathbf{k} \mathbf{d}-\mathbf{f}^{T} \mathbf{d}, \Pi_{h}^{e}=-\frac{1}{2} \boldsymbol{\beta}^{T} \mathbf{H} \boldsymbol{\beta}+\boldsymbol{\beta}^{T} \mathbf{G} \mathbf{d}-\mathbf{f}^{T} \mathbf{d} .
$$

where

$$
\begin{gathered}
\mathbf{k}=\int_{A^{e}}\left(\mathbf{P},{ }_{r}^{T} \mathbf{P},{ }_{r}+\mathbf{P},{ }_{z}^{T} \mathbf{P},,_{z}-k^{2} \mathbf{P}^{T} \mathbf{P}\right) r d A, \mathbf{f}=\int_{\Gamma_{t}^{e}} \mathbf{N}^{T} \overline{t r} d \Gamma, \\
\mathbf{H}=\int_{\partial A^{e}}\left(n_{r} \mathbf{P},{ }_{r}+n_{z} \mathbf{P},,_{z}\right)^{T} \mathbf{P} r d \Gamma \text { and } \mathbf{G}=\int_{\partial A^{e}}\left(n_{r} \mathbf{P},{ }_{r}+n_{z} \mathbf{P},{ }_{z}\right)^{T} \mathbf{N} r d \Gamma .
\end{gathered}
$$

The conventional element matrix is $\mathbf{k}$. As $g$ and thus $\mathbf{P}$ satisfy (1), $\mathbf{H}$ and $\mathbf{G}$ can be computed by 
domain integration as:

$$
\mathbf{H}=\int_{A^{e}}\left(\mathbf{P},{ }_{r}^{T} \mathbf{P},,_{r}+\mathbf{P},{ }_{z}^{T} \mathbf{P},,_{z}-k^{2} \mathbf{P}^{T} \mathbf{P}\right) r d A, \mathbf{G}=\int_{A^{e}}\left(\mathbf{P}_{r}^{T} \mathbf{N},{ }_{r}+\mathbf{P}_{{ }_{z}}^{T} \mathbf{P},{ }_{z}-k^{2} \mathbf{P}^{T} \mathbf{N}\right) r d A .
$$

to avoid the asymmetry of $\mathbf{H}$ due to numerical integration error. As there is no continuity requirement posed on $g, \boldsymbol{\beta}$ for each element is independent of those for the others and variation of $\boldsymbol{\beta}$ in the hybrid functional leads to

$$
\mathbf{H} \boldsymbol{\beta}=\mathbf{G d} \text { or } \boldsymbol{\beta}=\mathrm{H}^{-1} \mathbf{G d}
$$

By condensing $\boldsymbol{\beta}$,

$$
\Pi_{h}^{e}=\frac{1}{2} \mathbf{d}^{T}\left(\mathbf{G}^{T} \mathbf{H}^{-1} \mathbf{G}\right) \mathbf{d}-\mathbf{f}^{T} \mathbf{d} .
$$

The bracketed matrix product at the right-hand side of the expression gives the element matrix of the hybrid element.

\section{DISCONTINUOUS SPHERICAL-WAVE MODES}

While the above section outlines the principle to formulate the hybrid elements, the shape functions or modes for the discontinuous $g$ devised from the spherical-wave solution for axial symmetric 4node and 8-node quadrilateral elements will be presented in this section. The spherical-wave solution is:

$$
\left\{\frac{\exp \left(i k \rho_{S}\right)}{\rho_{S}}\right\} \text { or }\left\{\frac{\cos \left(k \rho_{S}\right)}{\rho_{S}}, \frac{\sin \left(k \rho_{S}\right)}{\rho_{S}}\right\}
$$

where $\rho_{s}^{2}=r^{2}+\left(z-z_{s}\right)^{2}$ is the square of distance between the source point $\mathrm{S}\left(0, z_{s}\right)$ and $(r, z)$, see Figure 1.

To formulate rank sufficient four- and eight-node element using spherical-waves, a minimal of four and eight wave modes from two and four poles are required, respectively. When a four-node square element with its edges parallel to the $r$ - or $z$-axes is considered, the four spherical-wave modes passing through the element centre along the directions of the $r$-and $z$-axes would lead to a rank deficient element. On the other hand, a rank sufficient element can successfully be obtained by the four modes passing through the element centre along the element diagonals. Moreover, additional discontinuous modes often lead to poor element accuracy. These observations are similar to those of the four-node and eight-node plane elements formulated with discontinuous plane-wave and Bessel solution modes [6,7]. To generalize the successful plane-wave modes to axial symmetric quadrilateral elements, the poles of the spherical-waves are selected such that the wave modes are "equal-distant" or "equal-spaced" to be described below. 


\subsection{The "Equal-Distant" Modes}

For the "equal-distant" modes, the spherical-wave poles are picked by using opposing node pairs in the sense that the parametric coordinates of a node can be obtained by multiplying those of the other node in the same pair with -1. A pole along the $z$-axis is fixed by the equal-distant requirement from a node pair. Hence, the node pair would be on the same wavefront of the spherical-wave from the pole as seen in Figure 2(a).

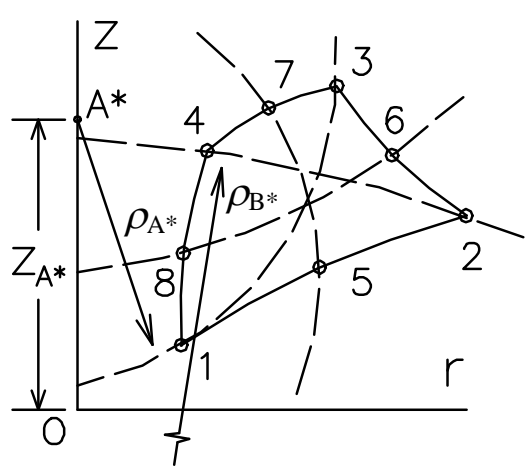

(a)

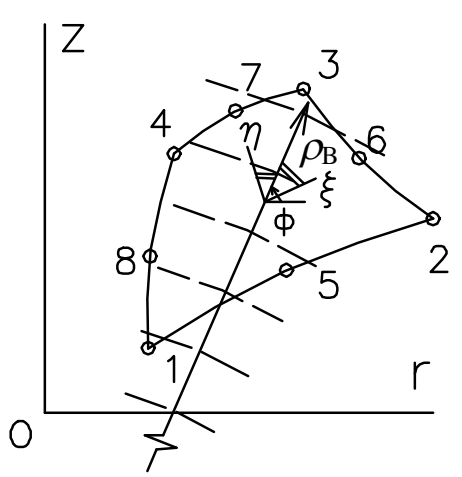

(b)

Figure 2. (a) Spherical-waves from poles equal-distant from opposing node pairs, e.g., $\left|A^{*} 1\right|=\left|A^{*} 3\right|$.

(b) The spherical-wave passes through the element origin at angle $\phi$ that bisects the $\xi$-and $\eta$-axes.

Four-Node Element SAQ4* - Considering node $1\left(r_{1}, z_{1}\right)$ and node $3\left(r_{3}, z_{3}\right)$ of the quadrilateral element in Figure 2(a). Parametric coordinates of the two nodes are $(-1,-1)$ and $(1,1)$. Hence, they are an opposing node pair. The $z$-coordinate $z_{A^{*}}$ of the pole $\mathrm{A}^{*}$ equal-distant from the two nodes is:

$$
z_{A^{*}}=\frac{r_{1}^{2}-r_{3}^{2}+z_{1}^{2}-z_{3}^{2}}{2\left(z_{1}-z_{3}\right)}
$$

Similarly, the coordinate $z_{B^{*}}$ of the pole $\mathrm{B}^{*}$ equal-distant from the nodes 2 and 4 is:

$$
z_{B^{*}}=\frac{r_{2}^{2}-r_{4}^{2}+z_{2}^{2}-z_{4}^{2}}{2\left(z_{2}-z_{4}\right)}
$$

The four-node element employing source points $\mathrm{A}^{*}$ and $\mathrm{B}^{*}$ and thus the following shape function matrix for $g$ :

$$
\mathbf{P}=\left[\frac{\bar{\rho}_{A^{*}} \cos \left(k \rho_{A^{*}}\right)}{\rho_{A^{*}}}, \frac{\bar{\rho}_{A^{*}} \sin \left(k \rho_{A^{*}}\right)}{\rho_{A^{*}}}, \frac{\bar{\rho}_{B^{*}} \cos \left(k \rho_{B^{*}}\right)}{\rho_{B^{*}}}, \frac{\bar{\rho}_{B^{*}} \sin \left(k \rho_{B^{*}}\right)}{\rho_{B^{*}}}\right]
$$

will be termed as SAQ4*. For any pole $S\left(0, z_{S}\right), \rho_{S}^{2}=r^{2}+\left(z-z_{S}\right)^{2}$ and $\bar{\rho}_{S}=\left.\rho_{S}\right|_{\xi=\eta=0}$ acts as a 
normalization factor for the related shape functions.

Eight-Node Element SAQ8* - For an eight-node element, four poles or eight wave modes are required for full rankness. Besides $\mathrm{A}^{*}$ and $\mathrm{B}^{*}$ used in $\mathrm{SAQ} 4^{*}$, nodes 5 and 7 define the pole $\mathrm{C}^{*}\left(0, z_{C}\right)$ whereas nodes 6 and 8 define the pole $\mathrm{D}^{*}\left(0, z_{D^{*}}\right)$. For $\mathrm{C}^{*}$ and $\mathrm{D}^{*}$,

$$
z_{C^{*}}=\frac{r_{5}^{2}-r_{7}^{2}+z_{5}^{2}-z_{7}^{2}}{2\left(z_{5}-z_{7}\right)}, z_{D^{*}}=\frac{r_{6}^{2}-r_{8}^{2}+z_{6}^{2}-z_{8}^{2}}{2\left(z_{6}-z_{8}\right)} \text {. }
$$

The eight-node element employing poles $\mathrm{A}^{*}$ to $\mathrm{D}^{*}$ and thus the following shape function matrix for $g:$

$$
\begin{gathered}
\mathbf{P}=\left[\frac{\bar{\rho}_{A^{*}} \cos \left(k \rho_{A^{*}}\right)}{\rho_{A^{*}}}, \frac{\bar{\rho}_{A^{*}} \sin \left(k \rho_{A^{*}}\right)}{\rho_{A^{*}}}, \frac{\bar{\rho}_{B^{*}} \cos \left(k \rho_{B^{*}}\right)}{\rho_{B^{*}}}, \frac{\bar{\rho}_{B^{*}} \sin \left(k \rho_{B^{*}}\right)}{\rho_{B^{*}}},\right. \\
\left.\frac{\bar{\rho}_{C^{*}} \cos \left(k \rho_{C^{*}}\right)}{\rho_{C^{*}}}, \frac{\bar{\rho}_{C^{*}} \sin \left(k \rho_{C^{*}}\right)}{\rho_{C^{*}}}, \frac{\bar{\rho}_{D^{*}} \cos \left(k \rho_{D^{*}}\right)}{\rho_{D^{*}}}, \frac{\bar{\rho}_{D^{*}} \sin \left(k \rho_{D^{*}}\right)}{\rho_{D^{*}}}\right]
\end{gathered}
$$

will be termed as SAQ8*.

\subsection{The "Equal-Spaced" Modes}

Equal-spaced modes have been employed in the six-node triangular elements by using the Fermat or Torricelli points inside the triangles [1,8]. For quadrilateral elements, Figure 2(b) depicts a spherical-wave passing through the element origin $\left(r_{0}, z_{0}\right)=\left.(r, z)\right|_{\xi=\eta=0}$ at an angle $\phi$ which bisects the $\xi$-and $\eta$-axes. Its pole $\mathrm{B}\left(0, z_{B}\right)$ can be determined from

$$
z_{B}=z_{0}-r_{0} \tan \phi
$$

For the "equal-spaced" modes, the other source points would be selected such that the directions of the waves passing through the element origin are equal-spaced.

Four-Node Element SAQ4 - The four-node element needs two source points for rank sufficiency. With the first direction fixed at $\phi$, the second direction should be taken as $\phi-\pi / 2$ which defines the pole $\mathrm{A}\left(0, z_{A}\right)$ with

$$
z_{A}=z_{0}-r_{0} \tan (\phi-\pi / 2)
$$

The hybrid element employing poles A and B will be termed as SAQ4. Its shape function matrix can be obtained from (14) by removing all the asterisks. 
Four-Node Element SAQ8 - The eight-node element needs four poles for rank sufficiency. With the first direction fixed at $\phi$, the other three equal-spaced directions should be taken as $\phi-\pi / 2, \phi$ $\pi / 4$ and $\phi+\pi / 4$ which define the source points $\mathrm{A}\left(0, z_{A}\right), \mathrm{C}\left(0, z_{C}\right)$ and $\mathrm{D}\left(0, z_{D}\right)$, respectively. It is trivial that

$$
z_{C}=z_{0}-r_{0} \tan (\phi-\pi / 4) \text { and } z_{D}=z_{0}-r_{0} \tan (\phi+\pi / 4)
$$

The hybrid element employing poles A and D will be termed as SAQ8. Its shape function matrix can be obtained from (16) by removing all the asterisks.

\subsection{Treatment for Distant Source Point and Singularity at the Source Point}

It may happen that a pole $S\left(0, z_{S}\right)$ is far away from the element within which the related wave is essentially parallel to the $z$-axis. To avoid numerical difficulty associated with the large radial distant " $\rho_{s}$ ", the two related spherical-wave modes would be replaced with the following plane-wave modes:

$$
\left\{\cos \left(k\left(z-\left.z\right|_{\xi=\eta=0}\right)\right), \sin \left(k\left(z-\left.z\right|_{\xi=\eta=0}\right)\right)\right\} \quad \text { if } \quad\left|\frac{\left.r\right|_{\xi=\eta=0}}{\left.z\right|_{\xi=\eta=0}-z_{S}}\right|<\tan \chi
$$

where $\chi$ is the small angle and is here taken to be $\pi / 120$, rather arbitrarily.

The wave mode $\cos (k \rho) / \rho$ is singular at its pole where $\rho=0$. When the pole is too close to the element, the singularity may pose numerical difficulty. A simple remedy to avoid the problem is to replace the hybrid element with the conventional one when $r=0$ in one or more than one element node.

\section{NUMERICAL EXAMPLES}

In this section, the predictions of the afore-discussed axial symmetric quadrilateral elements are reported. The elements include:

- CAQ4 - the conventional four-node element, see Section 2.

- SAQ4* - the four-node hybrid element based on "equal-distant" modes, see Section 3.1.

- SAQ4 - the four-node hybrid based on "equal-spaced" modes, see Section 3.2.

- CAQ8 - the conventional eight-node element, see Section 2.

- SAQ8* - the eight-node hybrid element based on "equal-distant" modes, see Section 3.1.

- SAQ8 - the eight-node hybrid based on "equal-spaced" modes, see Section 3.2. 
Unless specified otherwise, all the four- and eight-node elements are evaluated by the $2 \times 2$ and $3 \times 3$ quadratures, respectively. To compare the accuracy of the elements, the normalized error and the relative error with respect to the error of the conventional element (CAQ4 or CAQ8) will be computed. They are

$$
\begin{aligned}
& \text { Normalized error }=\left(\frac{\int_{\Omega}\left(u-u^{\text {exact }}\right)^{2} d \Omega}{\int_{\Omega}\left(u^{\text {exact }}\right)^{2} d \Omega}\right)^{1 / 2}=\left(\frac{\sum_{e} \int_{\Omega^{e}}\left(u-u^{\text {exact }}\right)^{2} d \Omega}{\sum_{e} \int_{\Omega^{e}}\left(u^{\text {exact }}\right)^{2} d \Omega}\right)^{1 / 2}, \\
& \text { Relative error }=\left(\frac{\int_{\Omega}\left(u-u^{\text {exact }}\right)^{2} d \Omega}{\int_{\Omega}\left(u^{\mathrm{C}}-u^{\text {exact }}\right)^{2} d \Omega}\right)^{1 / 2}=\left(\frac{\sum_{e} \int_{\Omega^{e}}\left(u-u^{\text {exact }}\right)^{2} d \Omega}{\sum_{e} \int_{\Omega^{e}}\left(u^{\mathrm{C}}-u^{\text {exact }}\right)^{2} d \Omega}\right)^{1 / 2}
\end{aligned}
$$

where $u$ denotes the finite element prediction, $u^{\text {exact }}$ denotes the exact solution, $u^{\mathrm{C}}$ denotes the prediction of the conventional element. For the hybrid elements, $g$ is the prediction within the element domain and, thus, $u$ is replaced by $g$ in the error measures. In both error measures, the integrals are evaluated by the $2 \times 2$ and $3 \times 3$ quadratures for the four- and eight-node elements, respectively. It has been checked that higher order integration rules lead to practicably identical results. Furthermore, the number of nodal spacings per wavelength

$$
N_{n}=\text { wave length } / \text { nodal spacing }=2 \pi /(k h)
$$

will be specified. In the expression, $h$ denotes the nodal spacing. For conventional element models, many literatures recommend $N_{n}>10$ (see, e.g., [17] among others) which, however, can be lowered for higher order elements.

For square element geometry, SAQ4* and SAQ8* are identical to SAQ4 and SAQ8, respectively. In examples involving non-square elements, the predictions of SAQ4* and SAQ8* are essentially graphically indistinguishable from those of SAQ4 and SAQ8, respectively. For clarity and conciseness, only the results of SAQ4 and SAQ8 would be presented in the subsequent result plots.

\subsection{Condition Number versus Integration Order and Invariance}

The quadrilateral in Figure 3 is modelled as a single element. The lengths of the element edges are $\sqrt{ } 0.65, \sqrt{ } 1.37, \sqrt{ } 0.5$ and $\sqrt{ } 0.9$ which gives an average nodal spacing of $\sim 0.91$ and $\sim 0.45$ for the four- and eight-node elements, respectively. 


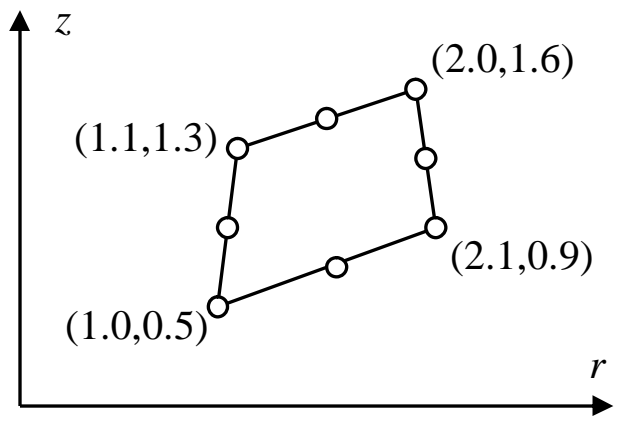

Figure 3. Condition numbers are computed for the portrayed element configuration.

For the four-node elements, $k=\pi / 5$ and $2 \pi / 5$ are considered. The relevant $N_{n}$ s are $\sim 10$ and $\sim 5$, respectively. Let $|\alpha|$ denote the eigenvalue magnitude of the element matrix, $|\alpha|_{\max },|\alpha|_{\min }$ and the condition number $N_{c}\left(=\left.|\alpha|_{\max }|| \alpha\right|_{\min }\right)$ are computed. Table 1 lists the results for CAQ4, SAQ4 and SAQ4* when $2 \times 2,3 \times 3$ and $4 \times 4$ quadradures are employed. Restricting to three significant figures, $N_{c} \mathrm{~s}$ of the elements do not change with respect to the integration orders. Moreover, CAQ4 is rank deficient when the $1 \times 1$ quadrature is used.

Table 1. $|\alpha|_{\max },|\alpha|_{\min }$ and $N_{c}$ of four-node elements under different quadratures.

\begin{tabular}{|c|c|c|c|c|c|c|c|c|c|c|}
\hline \multicolumn{2}{|c|}{} & \multicolumn{3}{c|}{$2 \times 2$} & \multicolumn{3}{c|}{$3 \times 3$} & \multicolumn{3}{c|}{$4 \times 4$} \\
\cline { 2 - 11 } & $|\alpha|_{\max }$ & $|\alpha|_{\min }$ & $N_{c}$ & $|\alpha|_{\max }$ & $|\alpha|_{\min }$ & $N_{c}$ & $|\alpha|_{\max }$ & $|\alpha|_{\min }$ & $N_{c}$ \\
\hline \multirow{3}{*}{ CAQ4 } & $k=\pi / 5$ & 15.84 & 0.72 & 22.0 & 15.84 & 0.72 & 22.0 & 15.84 & 0.72 & 22.0 \\
\cline { 2 - 11 } & $k=2 \pi / 5$ & 15.10 & 2.89 & 5.23 & 15.10 & 2.89 & 5.23 & 15.10 & 2.89 & 5.23 \\
\hline \multirow{2}{*}{ SAQ4 } & $k=\pi / 5$ & 15.66 & 0.73 & 21.4 & 15.66 & 0.73 & 21.4 & 15.66 & 0.73 & 21.4 \\
\cline { 2 - 12 } & $k=2 \pi / 5$ & 14.88 & 3.08 & 4.82 & 14.85 & 3.07 & 4.83 & 14.85 & 3.07 & 4.83 \\
\hline \multirow{2}{*}{ SAQ4 } & $k=\pi / 5$ & 15.68 & 0.73 & 21.5 & 15.68 & 0.73 & 21.5 & 15.68 & 0.73 & 21.5 \\
\cline { 2 - 11 } & $k=2 \pi / 5$ & 14.93 & 3.05 & 4.89 & 14.91 & 3.05 & 4.89 & 14.91 & 3.05 & 4.89 \\
\hline
\end{tabular}

For the eight-node elements, $k=2 \pi / 5$ and $4 \pi / 5$ are considered. The relevant $N_{n}$ s remain to be $\sim 10$ and $\sim 5$, respectively. Table 2 lists the computed $|\alpha|_{\max },|\alpha|_{\min }$ and $N_{c} \mathrm{~s}$ for the elements when $2 \times 2$, $3 \times 3$ and $4 \times 4$ quadradures are employed. $N_{c}$ of CAQ8 is least sensitive to the integration order. However, it is noted that the errors of CAQ8 evaluated by $2 \times 2$ and $3 \times 3$ quadratures are in the ratio of 2:1 which is similar to that of the plane conventional Q8 element [7]. Obviously, $2 \times 2$ quadradure is not adequate for the hybrid elements. $N_{c}$ s of the hybrid elements do not change when $3 \times 3$ quadrature is switched to $4 \times 4$ quadrature for $k=2 \pi / 5$ or $N_{n} \sim 10$. In the subsequent sub-sections, the results are computed by using $2 \times 2$ and $3 \times 3$ quadratures for all the four-node and eight-node 
elements, respectively. The integration orders are adequate for $N_{n} \sim 10$. As $N_{c}$ 's of all hybrid elements are finite, they are rank sufficient.

Table 2. $|\alpha|_{\max },|\alpha|_{\min }$ and $N_{c}$ of eight-node elements under different quadratures.

\begin{tabular}{|l|l|c|c|c|c|c|c|c|c|c|}
\hline \multicolumn{2}{|c|}{} & \multicolumn{3}{c|}{$2 \times 2$} & \multicolumn{3}{c|}{$3 \times 3$} & \multicolumn{3}{c|}{$4 \times 4$} \\
\cline { 3 - 11 } \multicolumn{2}{|c|}{} & $|\alpha|_{\max }$ & $|\alpha|_{\min }$ & $N_{c}$ & $|\alpha|_{\max }$ & $|\alpha|_{\min }$ & $N_{c}$ & $|\alpha|_{\max }$ & $|\alpha|_{\min }$ & $N_{c}$ \\
\hline \multirow{3}{*}{ CAQ8 } & $k=2 \pi / 5$ & 62.36 & 1.58 & 39.4 & 61.44 & 1.59 & 38.7 & 61.44 & 1.59 & 38.7 \\
\cline { 2 - 12 } & $k=4 \pi / 5$ & 54.16 & 1.12 & 48.3 & 50.76 & 1.18 & 43.1 & 50.76 & 1.18 & 43.1 \\
\hline \multirow{3}{*}{ SAQ8 } & $k=2 \pi / 5$ & 62.37 & 1.58 & 39.4 & 27.33 & 1.61 & 17.0 & 27.33 & 1.61 & 17.0 \\
\cline { 2 - 12 } & $k=4 \pi / 5$ & 42.76 & 1.79 & 23.9 & 25.21 & 0.90 & 28.1 & 25.41 & 0.93 & 27.3 \\
\hline \multirow{2}{*}{ SAQ8* } & $k=2 \pi / 5$ & 61.80 & 1.58 & 39.0 & 27.59 & 1.61 & 17.2 & 27.58 & 1.61 & 17.2 \\
\cline { 2 - 11 } & $k=4 \pi / 5$ & 45.70 & 1.74 & 26.3 & 25.57 & 0.92 & 27.9 & 25.83 & 0.95 & 27.1 \\
\hline
\end{tabular}

Different nodal connectivity orders which define the directions of the parametric axes are also attempted. The elements are also shifted along the z-direction. The computed $|\alpha|_{\max },|\alpha|_{\min }$ and $N_{c} \mathrm{~s}$ do not vary with the connectivity and the rigid body movement. Thus, the elements are invariant $[18,19]$.

\subsection{Plane-Wave thru Square Annular}

Figure 4 depicts a square annular with centers $(L, 0)$ and size length $L$ where $L=2$. The domain boundary is prescribed with the natural boundary condition derived from the following plane-wave solution:

$$
u=\cos (k z)
$$

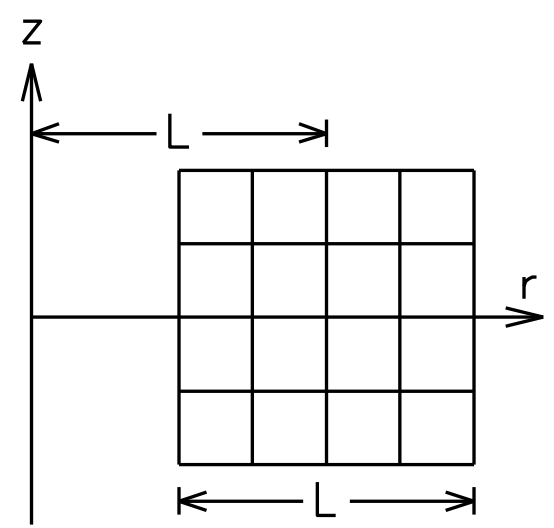

Figure 4. The $L \times L$ square annular with mean radius $L$ where $L=2$. Boundary conditions derived from the plane-wave solution $u=\cos (k z)$ or the cylindrical-wave solution $u=J_{0}(k r)$ are prescribed.

For the four-node elements, the convergence plots for $k L=20$ and 40 are shown in Figure 5 for 
$N_{n} \in[2 \pi, 32 \pi]$, respectively. For the same $N_{n}$, errors of the same element model at the two different $k L$ values are close. With $N_{n}=32 \pi$ and $k L=20$, the normalized error of CAQ4 is $2.5 \times 10^{-3}$ and the relative error of SAQ4 is $50 \%$. With $N_{n}=32 \pi$ and $k L=40$, the normalized error of CAQ4 is $2.3 \times$ $10^{-3}$ and the relative error of SAQ4 is again $50 \%$.

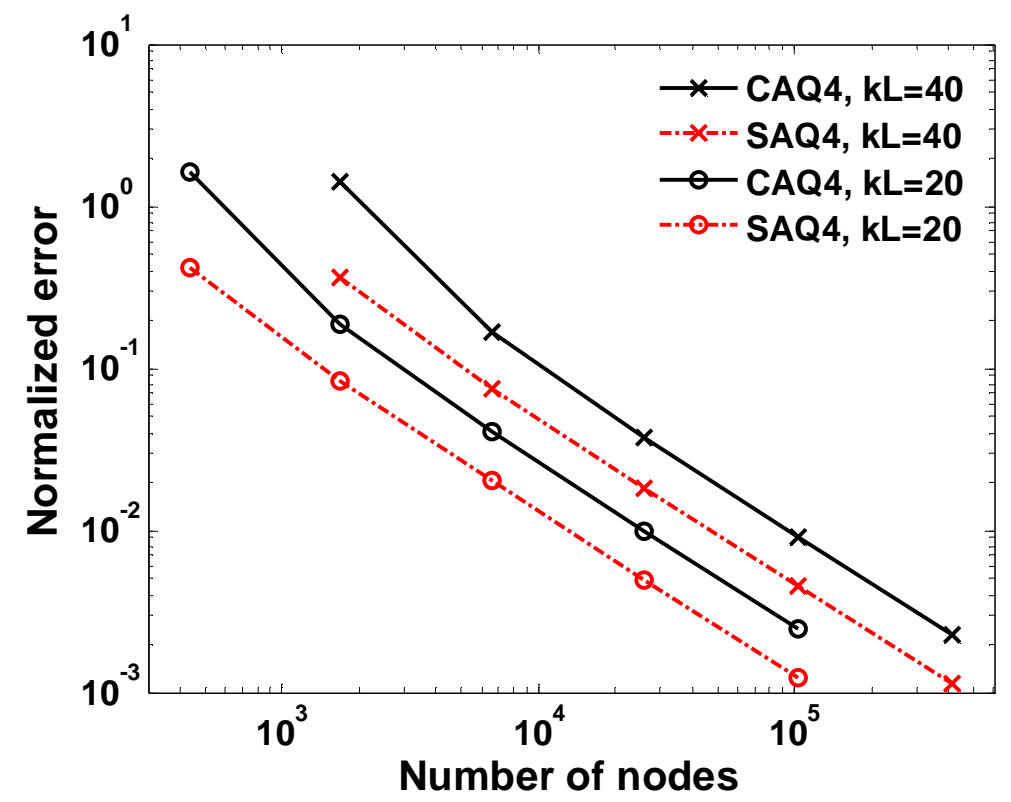

Figure 5. Errors of the four-node elements in the "plane-wave thru square annular" problem. $N_{n} \in$ $[2 \pi, 32 \pi]$. The relative error of SAQ 4 at $N_{n}=32 \pi$ are $50 \%$ for both $k L=20$ and 40 .

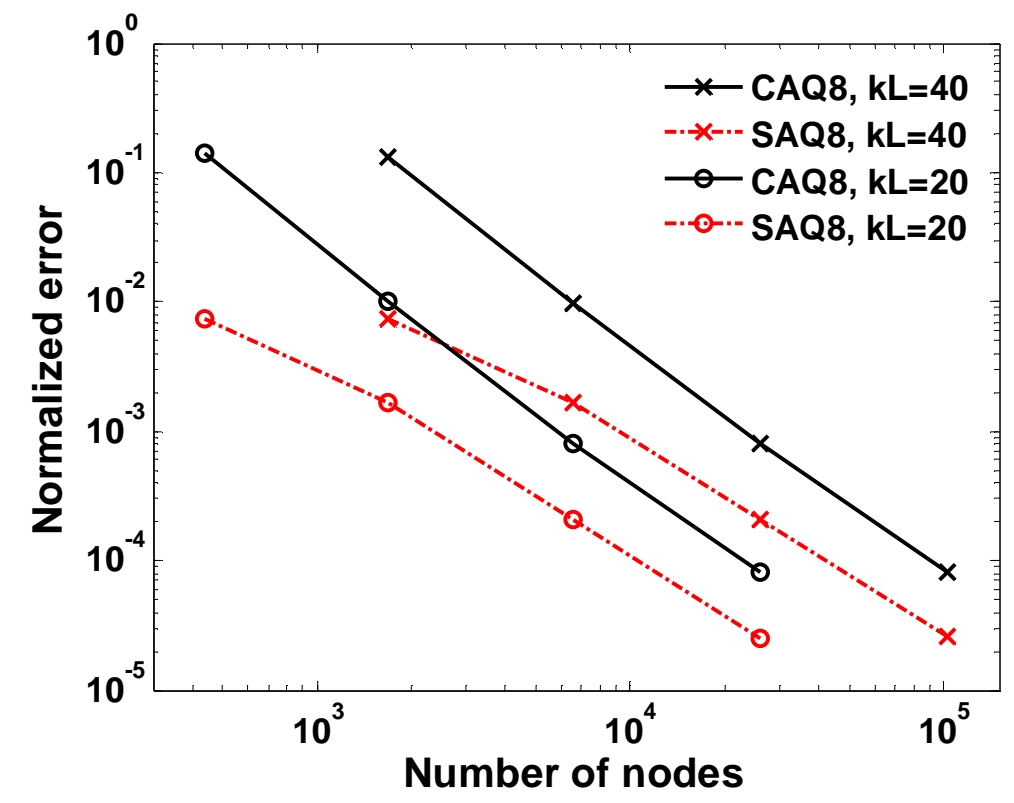

Figure 6. Errors of the eight-node elements in the "plane-wave thru square annular" problem. $N_{n} \in$ $[2 \pi, 16 \pi]$. At $N_{n}=16 \pi$, the relative error of SAQ8 are $32 \%$ for both $k L=20$ and 40 .

For the eight-node elements, the convergence plots for $k L=20$ and 40 are shown in Figure 6 for 
$N_{n} \in[2 \pi, 16 \pi]$, respectively. For the same $N_{n}$, errors of the same element model at the two different $k L$ values are close. With $N_{n}=16 \pi$ and $k L=20$, the normalized error of CAQ8 is $8.0 \times 10^{-5}$ and the relative error of SAQ8 is $32 \%$. With $N_{n}=16 \pi$ and $k L=40$, the normalized error of CAQ8 is $8.2 \times$ $10^{-5}$ and the relative error of SAQ8 is again $32 \%$. The convergence rate of the conventional and hybrid models are essentially the same.

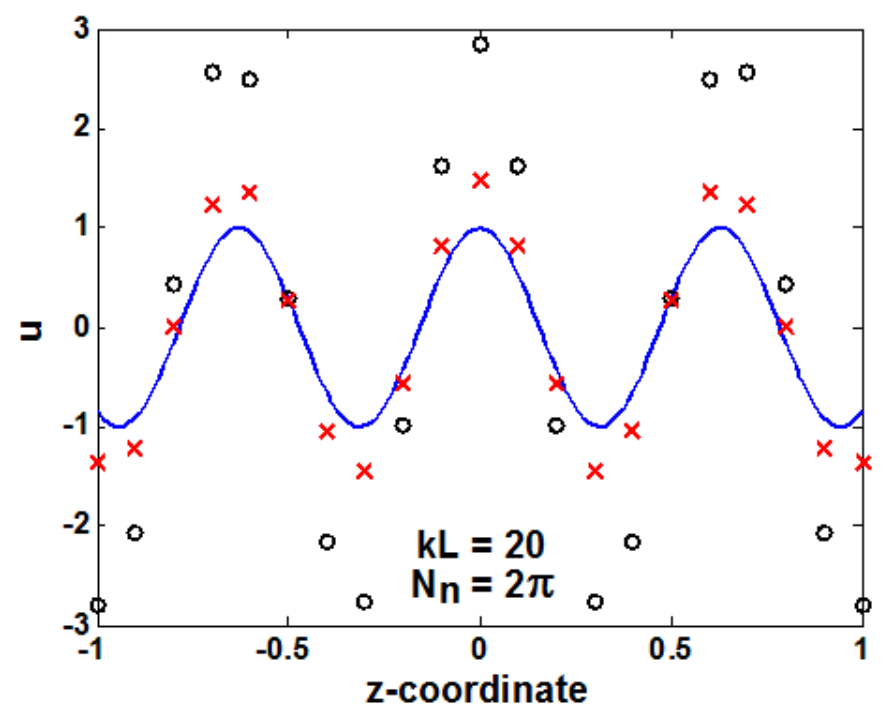

Figure 7. Predictions of CAQ4 (o) and SAQ4 ( $\times$ ) along $r=L$ in the "plane-wave thru square annular" problem with $k L=20$ and $N_{n}=2 \pi$. - denotes the exact solution.

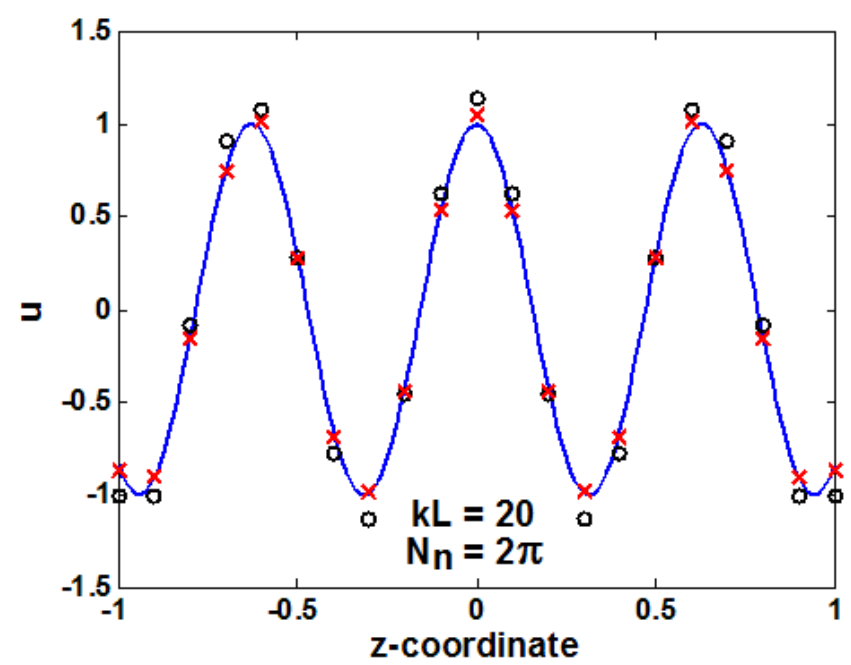

Figure 8. Predictions of CAQ8 (o) and SAQ8 $(\times)$ along $r=L$ in the "plane-wave thru square annular" problem with $k L=20$ and $N_{n}=2 \pi$. - denotes the exact solution.

Figures 7 and 8 plot the predictions of the four- and eight-node elements along the longitudinal centerline of the mesh $(r=L)$ for $k L=20$ and $N_{n}=2 \pi$. The errors echo those reported in Figures 5 and 6 . It can also be seen that the numerical wavenumbers, which are the wavenumbers constructed from the numerical predictions [19], of the eight-node elements are closer to the exact wavenumber 
that those of the four-node elements.

\subsection{Cylindrical-Wave thru Square Annular}

In this subsection, the boundary of the square annular in Figure 4 is prescribed with the natural boundary conditions derived from the following cylindrical-wave solution:

$$
u=J_{0}(k r)
$$

where $J_{0}$ is the zeroth order Bessel function of the first kind.

For the four-node elements, the convergence plots for $k L=20$ and 40 are shown in Figure 9 for $N_{n} \in[2 \pi, 32 \pi]$, respectively. With $N_{n}=32 \pi$ and $k L=20$, the normalized error of CAQ4 is $2.4 \times 10^{-3}$ and the relative error of SAQ4 is $50 \%$. With $N_{n}=32 \pi$ and $k L=40$, the normalized error of CAQ4 increases to $7.6 \times 10^{-3}$ and the relative error of SAQ4 is again $50 \%$.

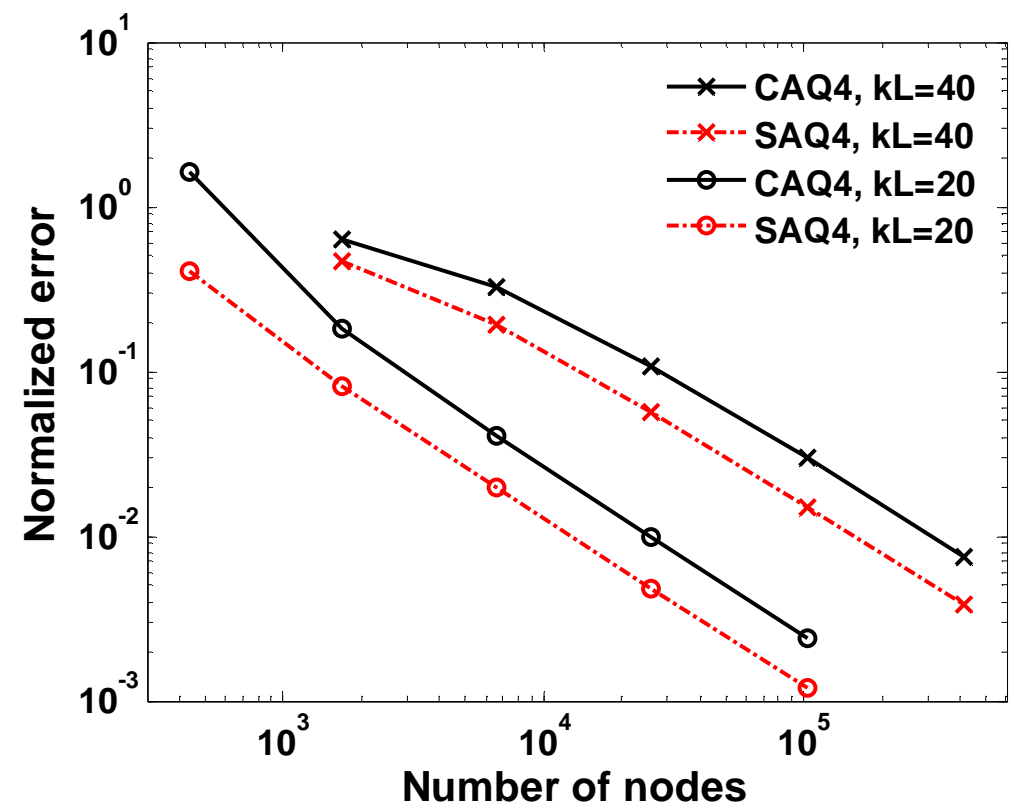

Figure 9. Errors of the four-node elements in the "cylindrical-wave thru square annular" problem. $N_{n}$ $\in[2 \pi, 32 \pi]$. At $N_{n}=32 \pi$, the relative errors of SAQ4 are $50 \%$ for both $k L=20$ and 40 .

For the eight-node elements, the convergence plots for $k L=20$ and 40 are shown in Figure 10 for $N_{n} \in[2 \pi, 16 \pi]$, respectively. With $N_{n}=16 \pi$ and $k L=20$, the normalized error of CAQ8 is $8.1 \times 10^{-5}$ and the relative error of SAQ8 is $32 \%$. With $N_{n}=16 \pi$ and $k L=40$, the normalized error of CAQ8 increases to $1.5 \times 10^{-4}$ and the relative error of SAQ8 is $22 \%$. At high nodal density, the convergence rate of the conventional and hybrid models are essentially the same. 


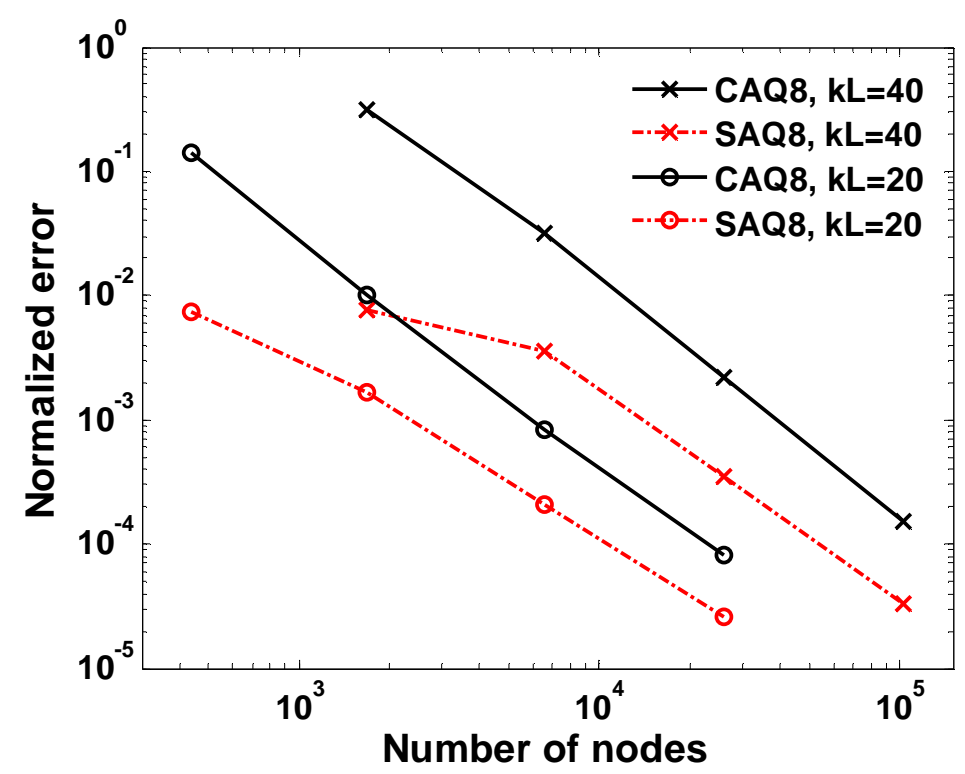

Figure 10. Errors of the eight-node elements in the "cylindrical-wave thru square annular" problem. $N_{n} \in[2 \pi, 16 \pi]$. At $N_{n}=16 \pi$, the relative errors of SAQ8 are $32 \%$ for $k L=20$ and $22 \%$ for $k L=40$.

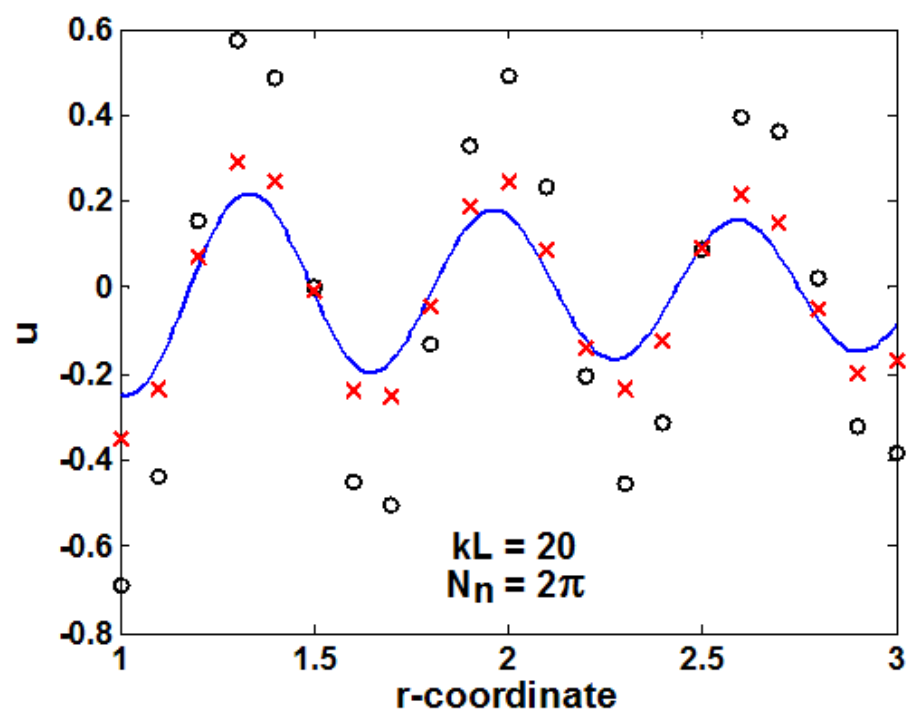

Figure 11. Predictions of CAQ4 (o) and SAQ4 $(x)$ along $z=0$ in the "cylindrical-wave thru square annular" problem with $k L=20$ and $N_{n}=2 \pi$. - denotes the exact solution.

Figures 11 and 12 plot the predictions of the four- and eight-node elements along the radial centerline of the mesh $(z=0)$ for $k L=20$ and $N_{n}=2 \pi$. The errors echo those reported in Figures 9 and 10. Again, the numerical wavenumbers of the eight-node elements are closer to the exact wavenumber that those of the four-node elements. 


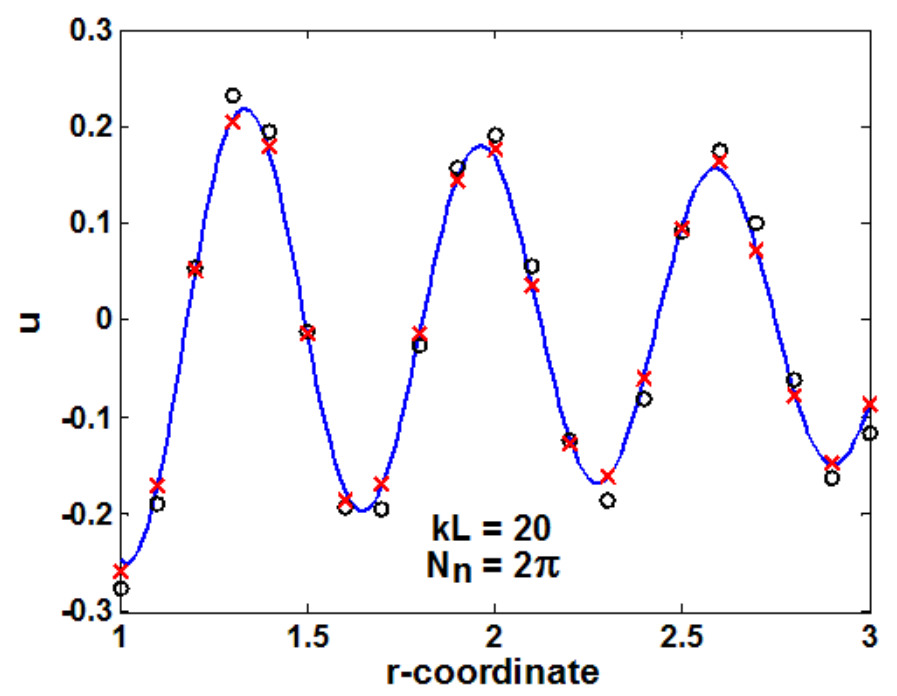

Figure 12. Predictions of CAQ8 (o) and SAQ8 $(\times)$ along $z=0$ in the "cylindrical-wave thru square annular" problem with $k L=20$ and $N_{n}=2 \pi$. - denotes the exact solution.

\subsection{Spherical-Wave thru Hemisphere}

Figure 13 depicts a hemispherical domain with radius $R=1$ partitioned into 48 elements. Boundary conditions corresponding to the spherical-wave solution:

$$
u=\frac{\sin \left(k \sqrt{r^{2}+z^{2}}\right)}{k \sqrt{r^{2}+z^{2}}}
$$

are implemented. The prescribed boundary conditions include $u=1$ at $(0,0), u,_{r}=0$ along $r=0$, $u,_{z}=0$ along $z=0$ and $u=\sin (k R) /(k R)$ along $r^{2}+z^{2}-R^{2}=0$.

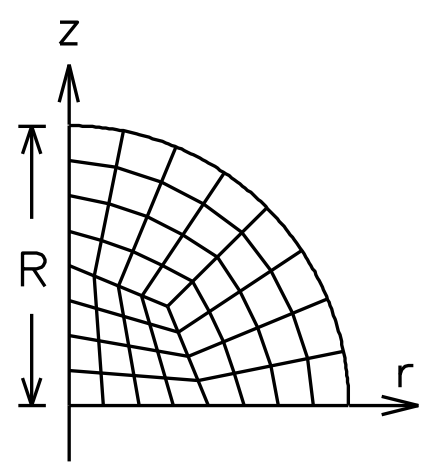

Figure 13. A semi-spherical domain with radius $R=1$ is partitioned into 48 elements. Boundary conditions derived from the spherical-wave solution $u=\sin \left[k \sqrt{ }\left(r^{2}+z^{2}\right)\right] /\left[k \sqrt{ }\left(r^{2}+z^{2}\right)\right]$ are prescribed.

For the four-node elements, the convergence plots for $k R=10$ and 20 are shown in Figure 14 for $N_{n} \in[2 \pi, 32 \pi]$ along the coordinate axes, respectively. With $N_{n}=32 \pi$ and $k R=10$, the normalized error of CAQ4 is $1.9 \times 10^{-3}$ and the relative error of SAQ4 is $51 \%$. With $N_{n}=32 \pi$ and $k R=20$, the normalized error of CAQ4 drops a bit to $1.7 \times 10^{-3}$ and the relative error of SAQ4 is $50 \%$. 


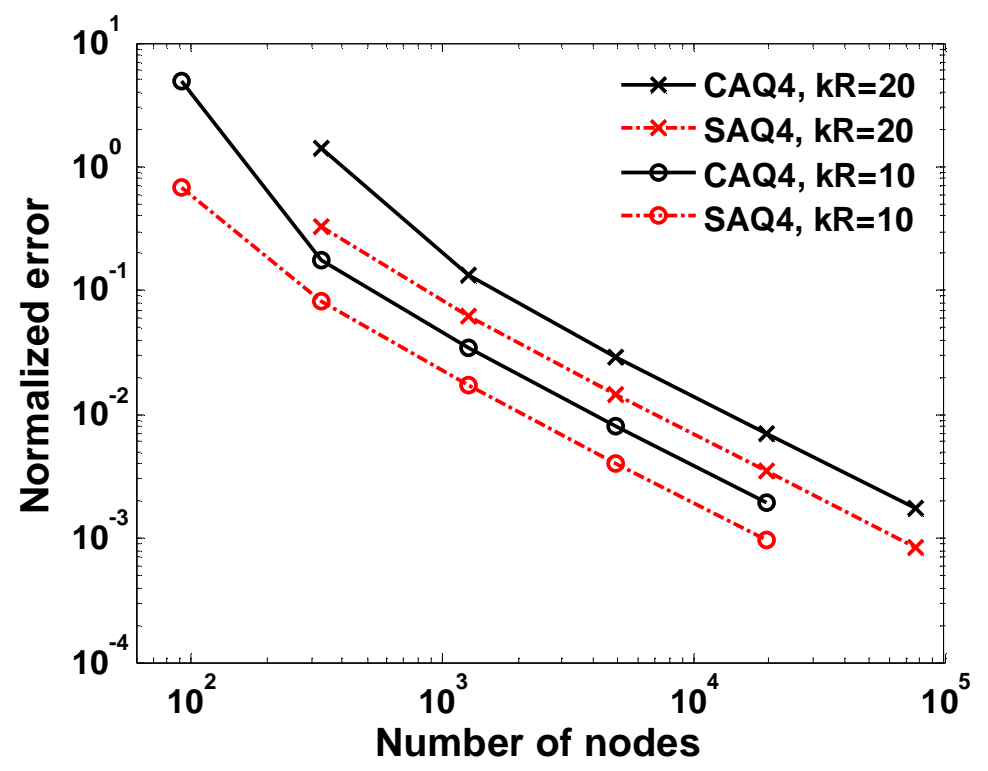

Figure 14. Errors of the four-node elements in the "spherical-wave thru hemisphere" problem. Along the coordinate axes, $N_{n} \in[2 \pi, 32 \pi]$. At $N_{n}=32 \pi$, the relative errors of SAQ4 are (a) $51 \%$ for $k R=10$ and $50 \%$ for $k R=20$.

For the eight-node elements, the convergence plots for $k R=10$ and 20 are shown in Figure 15 for $N_{n} \in[1.6 \pi, 12.8 \pi]$ along the coordinate axes, respectively. With $N_{n}=12.8 \pi$ and $k R=10$, the normalized error of CAQ8 drops a bit to $1.7 \times 10^{-4}$ and the relative error of SAQ8 is $34 \%$. With $N_{n}=$ $12.8 \pi$ and $k R=20$, the normalized error of CAQ8 is $1.5 \times 10^{-4}$ and the relative error of SAQ8 is $37 \%$. The convergence rate of the conventional and hybrid models are essentially the same.

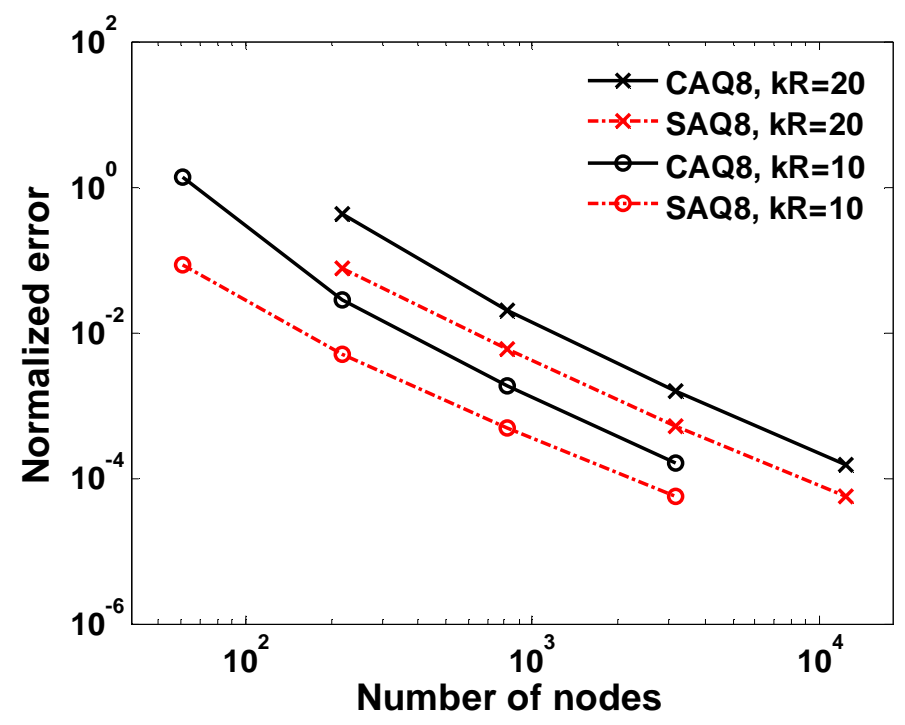

Figure 15. Errors of the eight-node elements in the "spherical-wave thru hemisphere" problem. Along the coordinate axes, $N_{n} \in[1.6 \pi, 12.8 \pi]$. The relative errors of SAQ8 at $N_{n}=12.8 \pi$ are $34 \%$ for $k R=10$ and $37 \%$ for $k R=20$. 


\subsection{Plane-Wave thru Rhombic Annular}

Figure 16 depicts a rhombic annular with centers $(L, 0)$ and size length $L$ where $L=2$. The essential boundary condition derived from the plane-wave solution in (23) is prescribed.

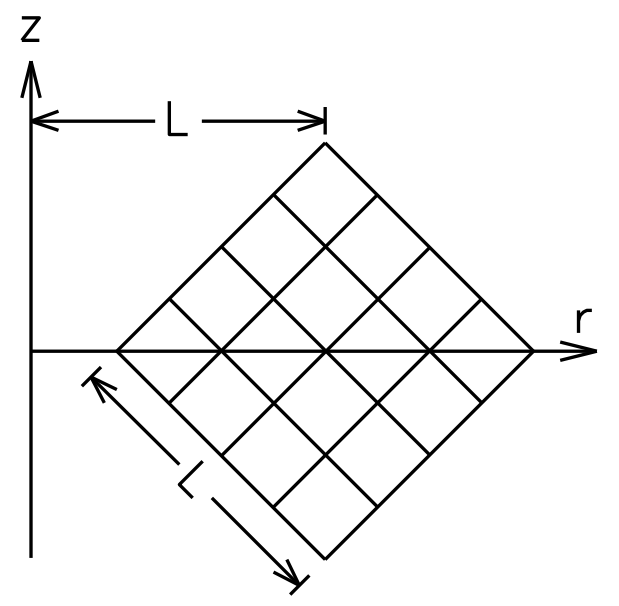

Figure 16. The $L \times L$ rhombic annular with mean radius $L$ where $L=2$. Boundary conditions derived from the plane-wave solution $u=\cos (k z)$, the cylindrical-wave solution $u=J_{0}(k r)$ or the sphericalwave solution $u=\sin \left[k \sqrt{ }\left(r^{2}+(z-L)^{2}\right)\right] / \sqrt{ }\left(r^{2}+(z-L)^{2}\right)$ are prescribed.

For the four-node elements, the convergence plots for $k L=20$ and 40 are shown in Figure 17 for $N_{n} \in[2 \pi, 32 \pi]$, respectively. With $N_{n}=32 \pi$ and $k L=20$, the normalized error of CAQ4 is $1.5 \times 10^{-3}$ and the relative error of SAQ4 is $11 \%$. With $k L=40$, CAQ4 does not converge monotonically. At $N_{n}=32 \pi$, the normalized error of CAQ4 increases substantially to $1.5 \times 10^{-1}$ and the relative error of SAQ4 is only $0.1 \%$.

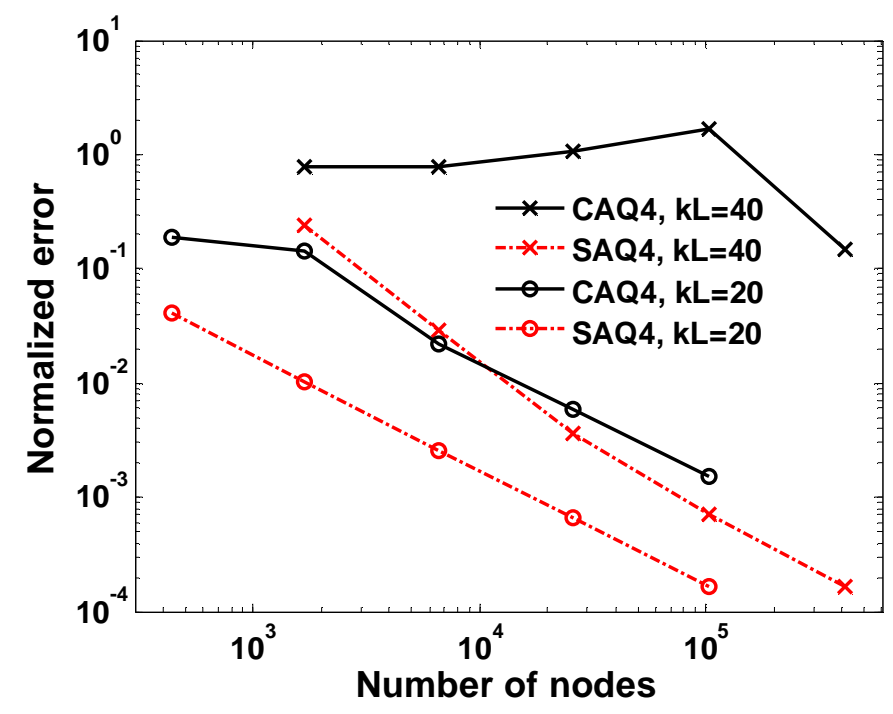

Figure 17. Errors of the four-node elements in the "plane-wave thru rhombic annular" problem. $N_{n} \in$ $[2 \pi, 32 \pi]$. At $N_{n}=32 \pi$, the relative errors of SAQ4 are $11 \%$ for $k L=20$ and $0.1 \% k L=40$. 
For the eight-node elements, the convergence plots for $k L=20$ and 40 are shown in Figure 18 for $N_{n} \in[2 \pi, 16 \pi]$, respectively. In both figures, relative error of SAQ8 is less $10 \%$ at the coarsest mesh. With $N_{n}=16 \pi$ and $k L=20$, the normalized error of CAQ8 is $4.0 \times 10^{-5}$ and the relative error of SAQ8 is $33 \%$. With $N_{n}=16 \pi$ and $k L=40$, the normalized error of CAQ8 increases to $1.0 \times 10^{-3}$ and the relative error of SAQ8 is $14 \%$. At high nodal density, the convergence rate of the conventional and hybrid models are essentially the same.

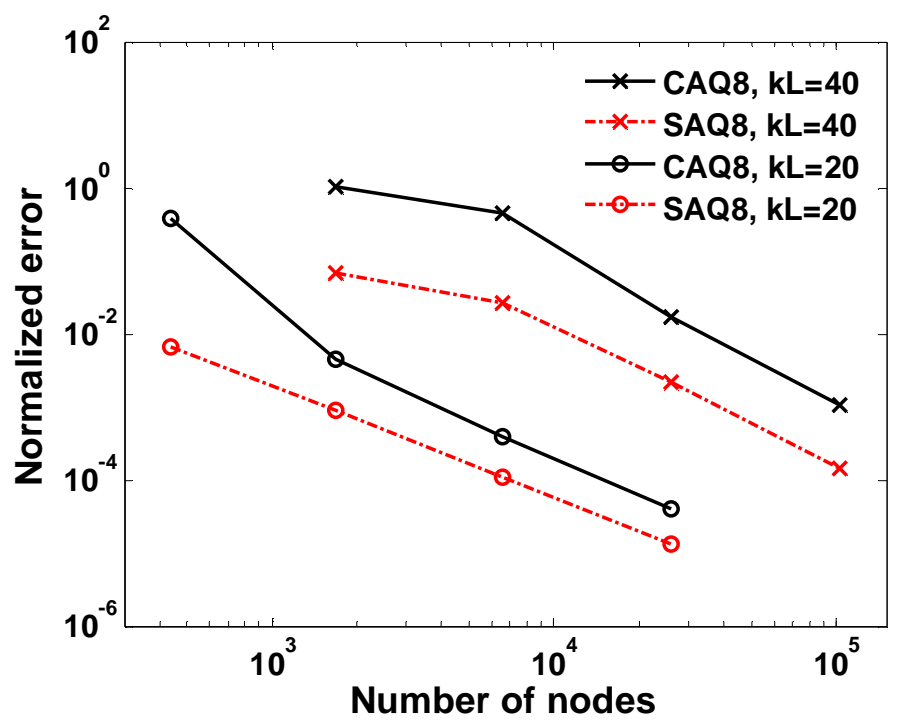

Figure 18. Errors of the eight-node elements in the "plane-wave thru rhombic annular" problem. $N_{n}$ $\in[2 \pi, 16 \pi]$. The relative errors of SAQ8 at $N_{n}=16 \pi$ are $33 \%$ for $k L=20$ and $14 \%$ for $k L=40$.

\subsection{Cylindrical-Wave thru Rhombic Annular}

The rhombic annular in Figure 16 is now prescribed with the essential boundary condition derived from the cylindrical-wave solution in (24).

For the four-node elements, the convergence plots for $k L=20$ and 40 are shown in Figure 19 for $N_{n} \in[2 \pi, 32 \pi]$, respectively. With $N_{n}=32 \pi$ and $k L=20$, the normalized error of CAQ4 is $4.4 \times 10^{-3}$ and the relative error of SAQ4 is $4.0 \%$. With $k L=40, \mathrm{CAQ} 4$ again does not converge monotonically. At $N_{n}=32 \pi$, the normalized error of CAQ4 is $9.3 \times 10^{-3}$ and the relative error of SAQ4 is only $2 \%$.

For the eight-node elements, the convergence plots for $k L=20$ and 40 are shown in Figure 20 for $N_{n} \in[2 \pi, 16 \pi]$, respectively. With $N_{n}=16 \pi$ and $k L=20$, the normalized error of CAQ8 is $5.3 \times 10^{-5}$ and the relative error of SAQ8 is $27 \%$. With $N_{n}=16 \pi$ and $k L=40$, the normalized error of CAQ8 is $7.4 \times 10^{-5}$ and the relative error of SAQ8 is $21 \%$. At high nodal density, the convergence rate of the conventional and hybrid models are essentially the same. 


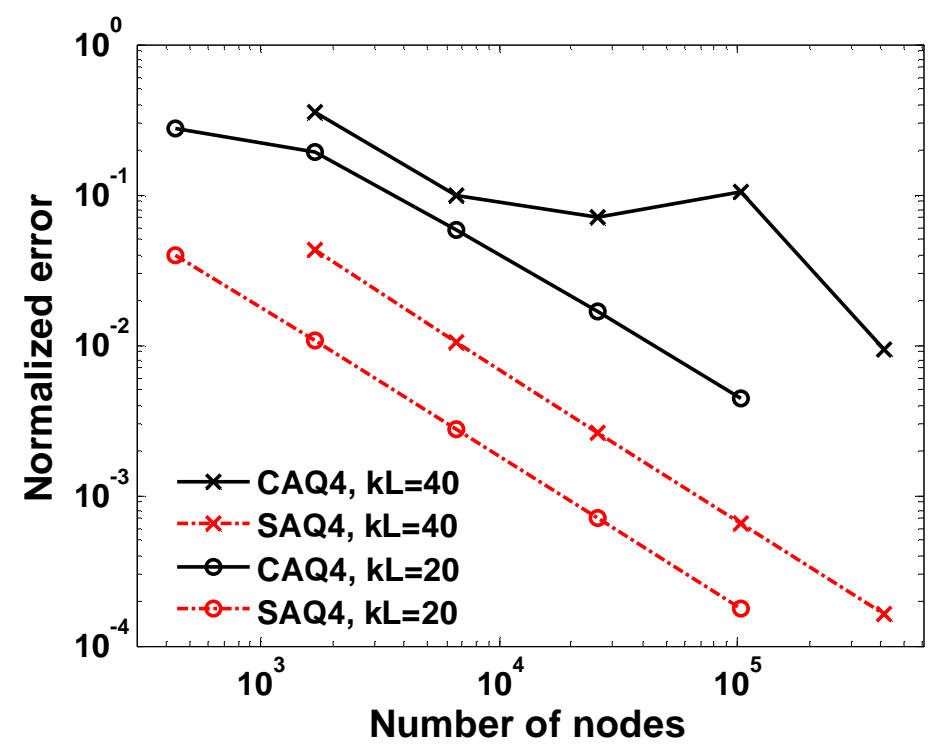

Figure 19. Errors of the four-node elements in the "cylindrical-wave thru rhombic annular" problem. $N_{n} \in[2 \pi, 32 \pi]$. At $N_{n}=32 \pi$, the relative errors of SAQ 4 are $4 \%$ for $k L=20$ and $2 \%$ for $k L=40$.

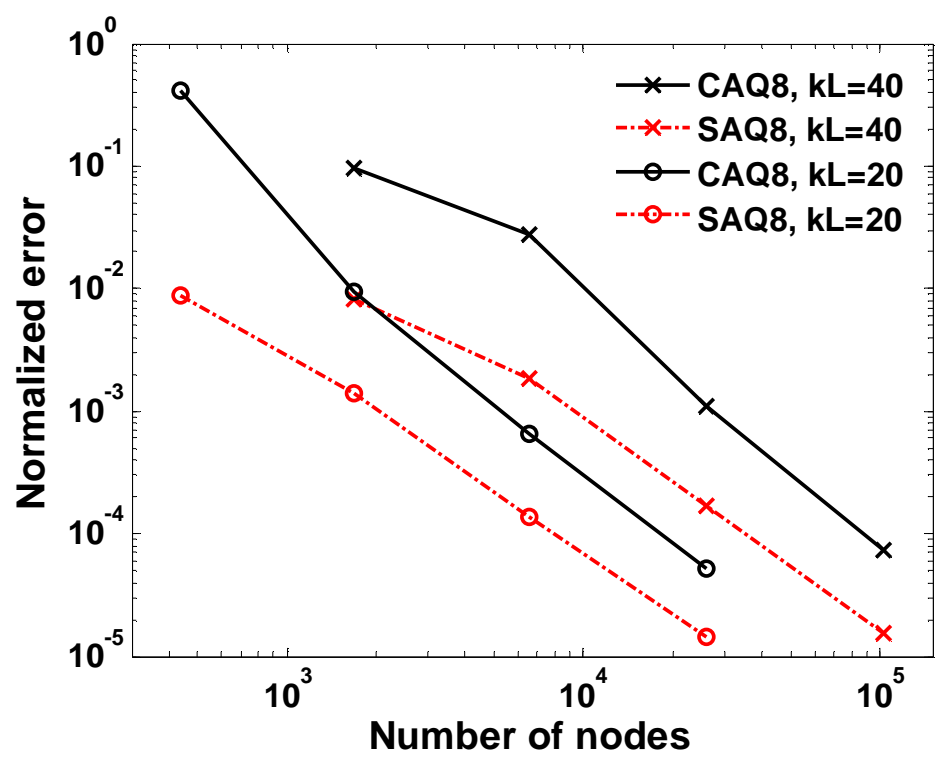

Figure 20. Errors of the eight-node elements in the "cylindrical-wave thru rhombic annular" problem. $N_{n} \in[2 \pi, 16 \pi]$. The relative errors of SAQ8 at $N_{n}=16 \pi$ are $27 \%$ for $k L=20$ and $21 \%$ for $k L=40$.

\subsection{Spherical-Wave thru Rhombic Annular}

The errors of SAQ4 in last two problems involving the rhombic annular domain (normalized error $\approx \sim 10^{-4}$ ) are nearly 10 times smaller than those involving the square annular (normalized error $\approx \sim 10^{-3}$ ) at the highest nodal density. Coincidently, the wave directions of the exact solutions are parallel or essentially parallel to those of the discontinuous modes inside the hybrid elements modelling the rhombic annular. To investigate whether the coincidence in the directions contribute to the higher accuracy, the rhombic annular are prescribed with essential boundary conditions according to the spherical-wave solution: 


$$
u=\frac{\sin \left(k \sqrt{r^{2}+(z-L)^{2}}\right)}{k \sqrt{r^{2}+(z-L)^{2}}}
$$

whose pole is $(0, L)$ and propagration directions are no more parallel to those of the discontinuous modes inside the elements. The convergence plots for $k L=20$ and 40 are shown in Figure 21 for $N_{n}$ $\in[2 \pi, 32 \pi]$, respectively. With $N_{n}=32 \pi$ and $k L=20$, the normalized error of CAQ4 is $2.8 \times 10^{-3}$ and the relative error of SAQ4 is $45 \%$. With $N_{n}=32 \pi$ and $k L=40$, the normalized error of CAQ4 is $6.1 \times 10^{-3}$ and the relative error of SAQ4 is $41 \%$. In both cases, the normalized errors of SAQ4 are $\sim 2 \times 10^{-3}$ which is considerably larger than those of the plane-wave and cylindrical-wave problems involving the rhombic annular but is similar to those of the same problems involving the square annular.

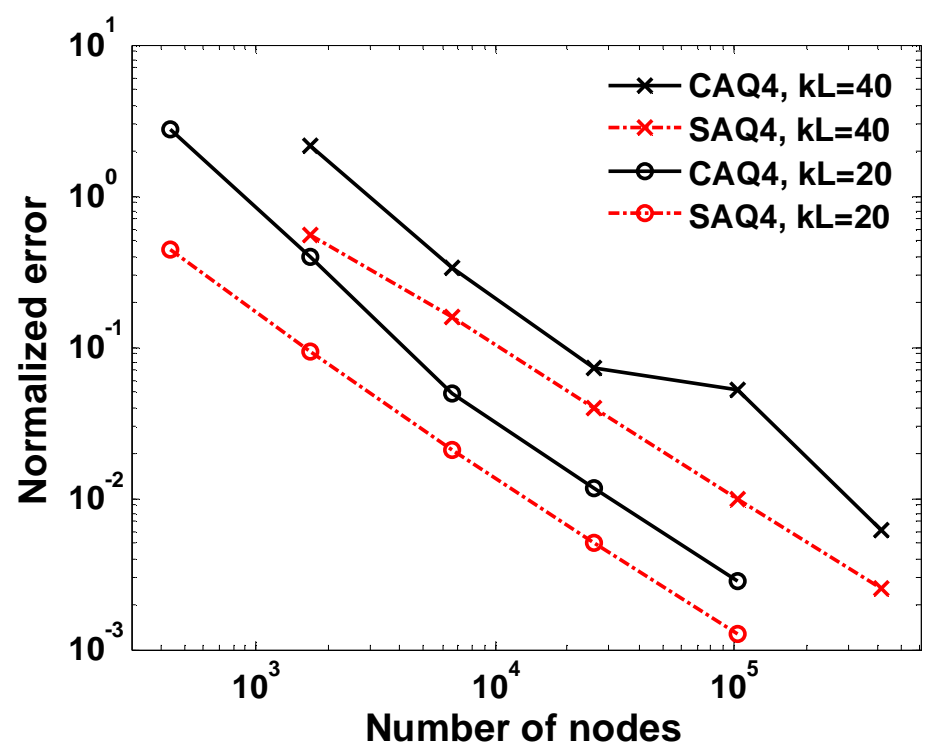

Figure 21. Errors of the four-node elements in the "spherical-wave with source $(0, L)$ thru rhombic annular" problem. $N_{n} \in[2 \pi, 32 \pi]$. The relative errors of SAQ4 at $N_{n}=32 \pi$ are $45 \%$ for $k L=20$ and $41 \%$ for $k L=40$.

\subsection{Wavenumber Sweep for the Four-Node Elements}

The coincidence of the wave directions in the exact solution and the discontinous modes of the four-node element model leads to the distinctive element accuracy in Sections 4.5 and 4.6. The observation is simlar to that of the four-node plane hybrid element in which its discontinous modes are the plane-wave modes along the element diagonals [7]. Another interesting observation is illustrated in Figures 22 and 23. Figures 22(a) and 22(b) show the wavenumber sweeps for the problems of plane-wave (see (23)) thru the square annular and spherical-wave (see (26)) thru the rhombic annuar with nodal spacing 1/80, respectively. The results are typical and contain multiple spikes due to fictitious resonanting predicitons. It can also be seen that the relative error of SAQ4, with respect to CAQ4, is roughly $50 \%$. On the other hand, Figure 23(a) shows the wavenumber 
sweeps for the problem of cylindrical-wave (see (24)) thru the rhombic annular whilst Figure 23(b) shows the wavenumber sweeps for the problem of plane-wave thru a plane square panel using the four-node plane conventional element CQ4 and the hybrid element PQ4 [7]. The same mesh with nodal spacing 1/80 is again employed and the exact wave directions are essentially and exactly parallel to those of the discontinuous modes of the axial symmetric and plane hybrid elements, respectively. Unlike the typical results portrayed in Figure 22 there is no fictitious resonanting prediciton or spike. Nevertheless, this interesting advantage is not generic and disappear when the directions of the exact solution and the discontinuous modes of the elements differ.

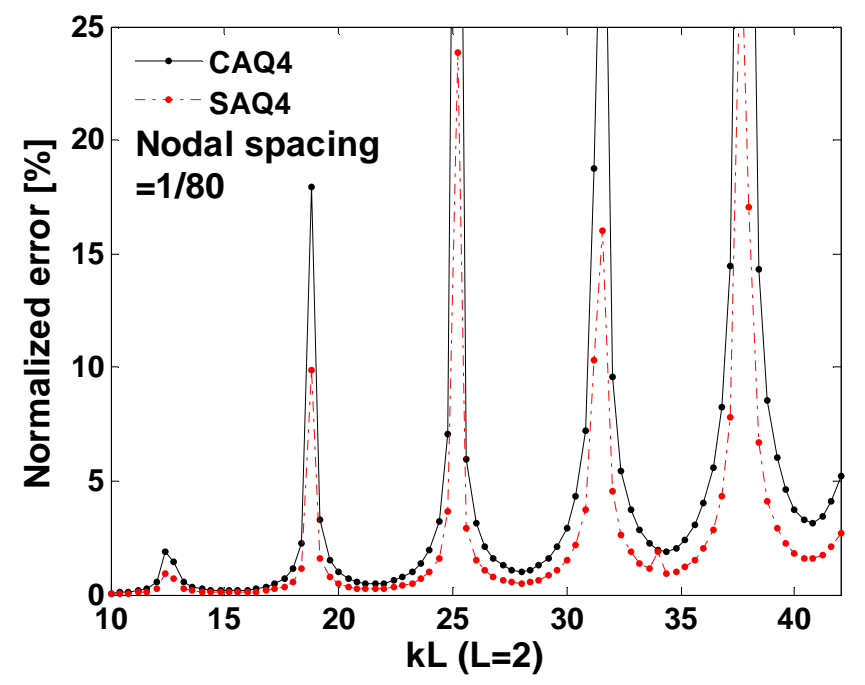

(a)

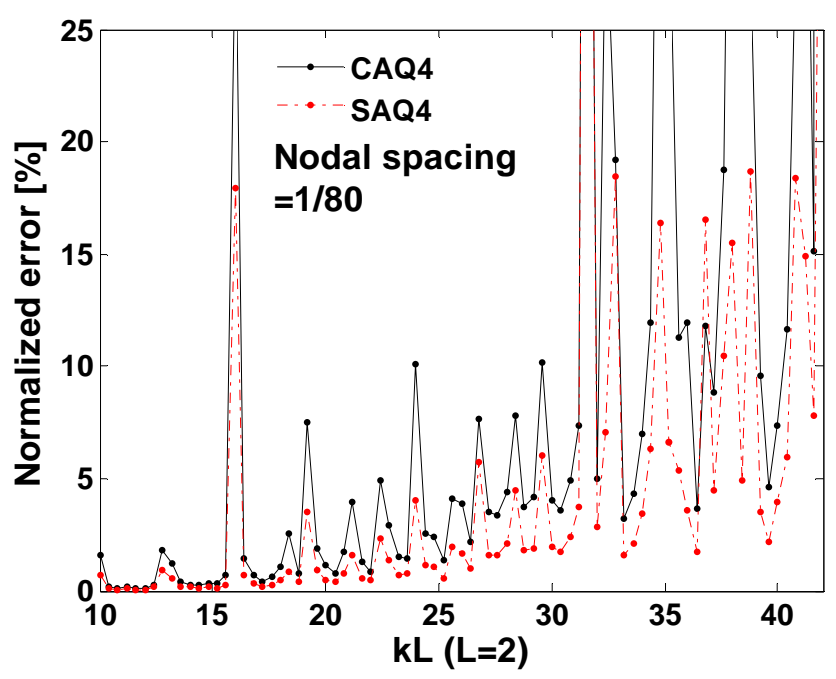

(b)

Figure 22. Errors of the four-node elements versus wavenumber in (a) the "plane-wave thru square annular" problem and (b) the "spherical-wave with source $(0, L)$ thru rhombic annular" problem. 


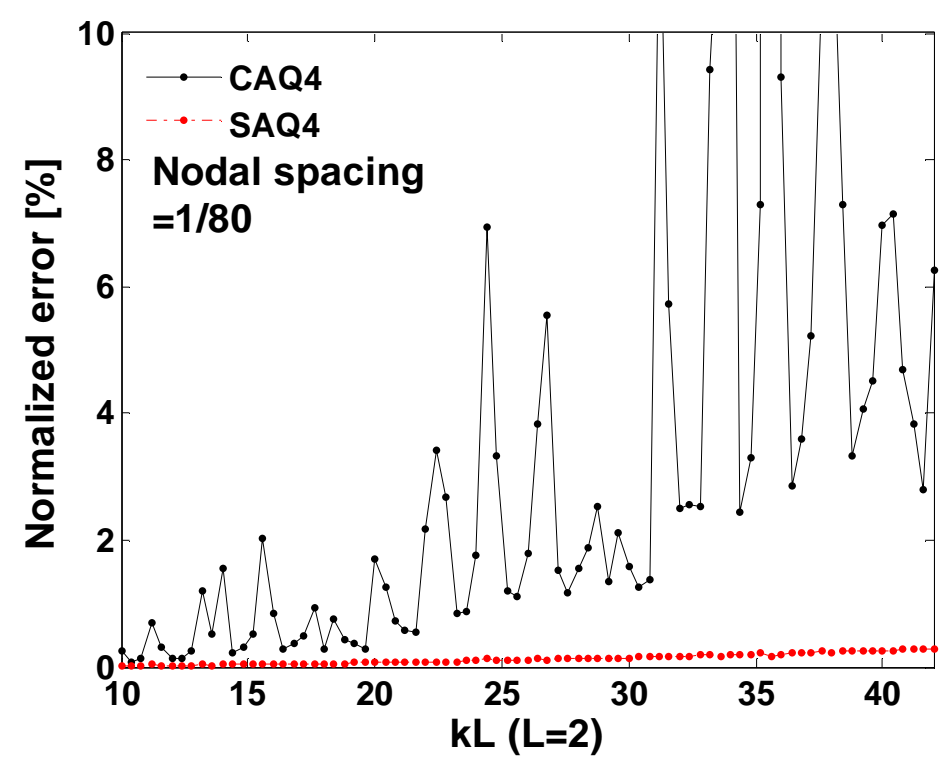

(a)

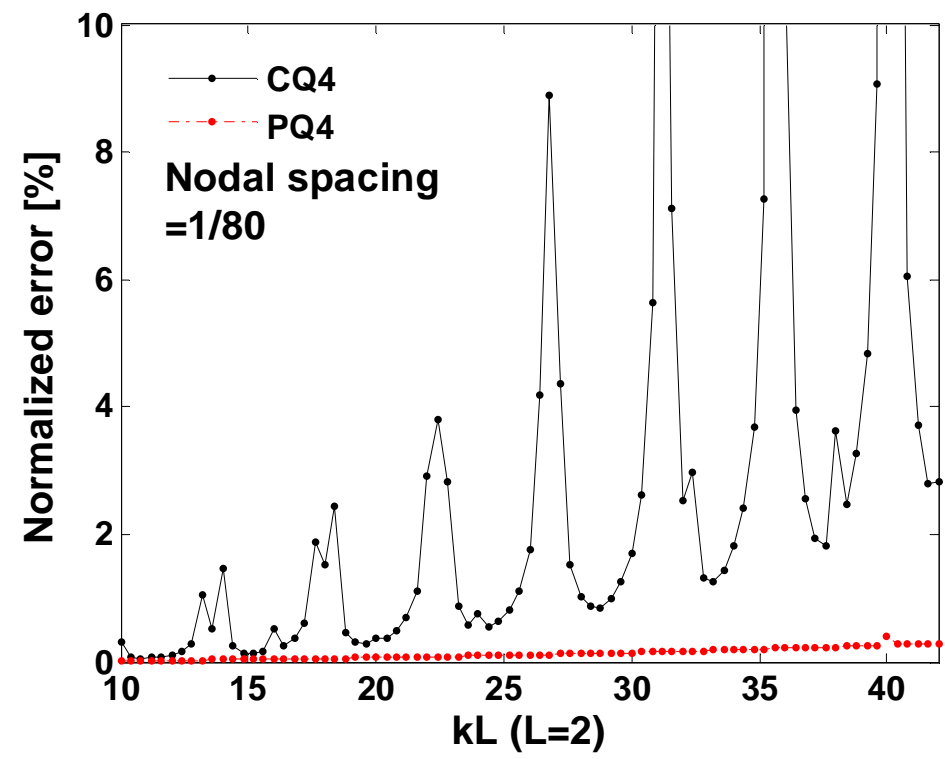

(b)

Figure 23. (a) Errors of the four-node axial symmetric elements versus wavenumber in the "cylindrical-wave thru rhombic annular" problem and (b) errors of the four-node plane elements versus wavenumber in the "plane-wave thru square panel" problem.

\section{CONCLUSION}

Four- and eight-node hybrid quadrilateral axial symmetric elements are devised in this paper by a hybrid variational functional that enforces the equality of a continuous and a discontinuous Helmholtz variables. The former is constructed by nodal interpolation and the latter is composed of spherical-waves with poles at the axis of symmetry. They have the same system dofs as the conventional or continuous Galerkin elements based on the single field variational functional and can 
readily be incorporated into the standard finite element program framework. Boundary conditions can also be prescribed as if they were conventional elements. Two ways of selecting the sphericalwave poles have been attempted and the resultant elements yield close predictions. With respect to four- and eight-node conventional elements, the four-and eight-node hybrid elements are typically $\sim 50 \%$ and $\sim 70 \%$ less erroneous, respectively. The CPU time for computing the element matrices of the hybrid elements are inevitably higher than that of the conventional elements. The gain in accuracy is a justifying factor and the other factor is that the total CPU time for a large problem is dominated by the solution time (for solving the system equation).

Acknowledgment - The support of Hong Kong Research Grant Council in the form of the GRF grant HKU 7167/08E is gratefully acknowledged. Majority of the work was completed when the second author was a postdoctoral fellow at the University of Hong Kong. 


\section{REFERENCES}

[1] Liu GH, Zhang QH, Sze KY (2011). Spherical-wave based triangular finite element models for axial symmetric Helmholtz problems. Finite Elements in Analysis \& Design 47: 342-350.

[2] Stojek M (1998). Least square Trefftz-type elements for the Helmholtz equation. Int J Numer Meth Engng. 41: 831-849.

[3] Stojek M, Markiewicz M, Mahrenholtz O (2000). Diffraction loads on multiple vertical cylinders with rectangular cross section by Trefftz-type finite elements, Computers \& Structures 75: 335-345.

[4] Freitas JAT, Cismaşiu C (2003). Hybrid-Trefftz displacement element for spectral analysis of bounded and unbounded media. Int.J.Solids \& Structures 40: 671-699.

[5] Pluymers B, Van Hal B, Vandepitte D, Desmet W (2007). Trefftz-based methods for timeharmonic acoustics. Arch Comput Methods Eng 14: 343-381.

[6] Sze KY, Cheung YK (2008). A hybrid-Trefftz finite element model for Helmholtz problem. Commun.Numer.Methods Engrg. 24: 2047-2060.

[7] Sze KY, Liu GH, Fan H (2010). Four- and eight-node hybrid-Trefftz quadrilateral finite element models. Computer Methods Applied Mechanics \& Engrg. 199: 598-614.

[8] Sze KY, Liu GH (2010). Hybrid-Trefftz six-node triangular finite element models for Helmholtz problem, Computational Mechanics 46: 455-470.

[9] Sze KY, Zhang QH, Liu GH (2011). Multi-field three-node triangular finite element models for Helmholtz Problem. J. Computational Acoustics, 19: 317-334.

[10] Melenk JM, Babuška I (1997). The partition of unity method. Int.J.Numer.Meth.Engng. 40:727 - 758 .

[11] Laghrouche O (2000), Bettess P. Short wave modelling using special finite elements. $J$. Computational Acoustics 8: 189-210.

[12] Ortiz P, Sanchez E (2001). An improved partition of unity finite element method for diffraction problems. Int.J.Numer.Meth.Engng. 50:2727 - 2740.

[13] Mohamed MS, Laghrouche O, El-Kacimi A (2010). Some numerical aspects of the PUFEM for efficient solution of 2D Helmholtz problems. Computers \& Structures 88: 1484-1491.

[14] Farhat C, Harari I, Hetmaniuk U. The discontinuous enrichment method for multiscale analysis. Computer Meth.in Appl.Mech. \& Engng. 192: 1389-1419 (2003)

[15] Farhat C, Harari I, Hetmaniuk U. A discontinuous Galerkin method with Lagrange multipliers for the solution of Helmholtz problems in the mid-frequency regime. Computer Meth.in Appl.Mech. \& Engng. 192: 3195-3209 (2003)

[16] Freitas JA, Almeida JP, Pereira EMB. Non-conventional formulations for the finite element method. Comput.Mech. 23: 488-501 (1999).

[17] Deraemaeker A, Babuška I, Bouillard P. Dispersion and pollution of the fem solution for the Helmholtz equation in one, two and three dimensions. Inter.J.Numer.Meth.Engng. 46: 471 499 (1999).

[18] Spilker RL, Maskeri SM, Kania E. Plane isoparametric hybrid-stress elements: invariance and optimal sampling. Int.J.Numer.Meth.Engng., 17, 1469-1496 (1981)

[19] Sze KY, Chow CL, Chen WJ. On invariance of isoparametric hybrid elements. Commun. Numer.Meth.Engng. 8: 385-406 (1992) 